\title{
Visual Presentation Challenges for Mobile Spatial Applications: Three Case Studies
}

\author{
Peter Fröhlich ${ }^{1}$, Matthias Baldauf ${ }^{1}$, Peter Reichl ${ }^{1}$, Robert F. Tobler ${ }^{2}$ \\ ${ }^{1}$ Telecommunications Research Center Vienna (ftw.) \\ ${ }^{2}$ VRVis Research Center, Vienna \\ \{froehlich | baldauf | reichl\}@ftw.at; tobler@vrvis.at
}

\begin{abstract}
Using mobile devices for exploring and interacting with the user's physical environment is among the most promising candidates for commercially successful future telecommunication services and applications. This trend is confirmed by the rollout of latest mobile phones equipped with advanced navigation features like built-in GPS receivers, digital compasses and acceleration sensors. The increasing technological potential of Mobile Spatial Interaction (MSI), however, requires a wider comprehension of appropriate visualization possibilities for spatially referenced content in the field of Mobile Human-Computer Interaction.

In this paper, we argue for an integrated approach comprising innovations in MSI with latest advances in visualization. In order to demonstrate the potential of this joint approach, we present a series of three related projects and highlight in each case research challenges and results in the field of Mobile HCI and mobile application development as well as corresponding advances of the underlying server-side rendering algorithms.
\end{abstract}

Keywords--- Mobile Spatial Interaction, Mobile HCI, Rendering, Visualization

\section{Introduction}

The idea of combining geo-spatial information with mobile communication technology is gaining increasing interest in academia and industry alike. Currently, new forms of georeferenced services, such as map-mashups [1] , are emerging almost on a daily basis.

Recent research projects are dealing with ways on how mobile devices can be used as an interface to this growing body of geo-spatial information, to enable direct access and interaction in the user's surroundings. The common denominator of these research endeavors is that they are dealing with 'Mobile Spatial Interaction' (MSI). MSI aims at creating a sense of orientation and direct mapping between the user's environment and related digital information. To this end, MSI goes far beyond the traditional notion of 'location-awareness' or 'locationbased services'.

A visionary concept important for this research was proposed by Egenhofer [2] in the late 1990ies. His ideas comprise 'Smart Compasses' which direct users to specific locations, 'Geo-Wands' that enable users to point at objects of interest to receive more information about them, and 'Smart Horizons' creating a virtual representation related to their current field of view (see Figure 1). Another early project paving the way for MSI was 'CyberGuide', a prototypical mobile location- and orientation-aware tour guide for visitors of Atlanta's Georgia Tech university campus [17] . Since then, MSI has experienced an enormous boost in terms of both technological and market potential, and it is by now strongly pushed by the mobile handset industry, as is clearly indicated by the current trend towards location-aware mobile phones with integrated compasses [7] .

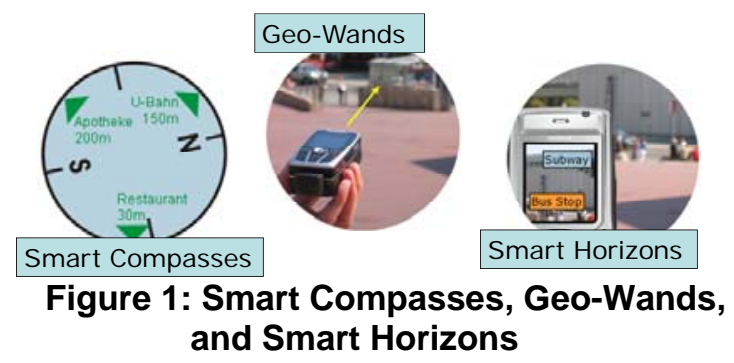

The primary application field currently targeted by industry is navigation and wayfinding for in-car and pedestrian use. A strong advantage of MSI is the very intuitive and direct access to content attached to the surroundings, so-called points of interest (POI). If, for example, someone is interested in the price of apartments that are going to be built at a certain construction site, one could imagine an application where she could immediately get this information onto her phone, simply by pointing at the spot of interest. Another advantage MSI offers is exploration by means of augmented reality. The capabilities of today's mobile technology for exploration have 
been demonstrated by Schall et al. [5] , who enabled field workers to maintain underground infrastructure that would otherwise not be visible.

Despite of such promising prospects, MSI still requires a great deal of applied research to make its applications truly acceptable for the mass market (cf [3] ). The manifold challenges to be faced range from purely engineering-related spatial sensing issues to rather economic topics, such as scalability of content provision or business modeling. Nevertheless, the 'sine qua non' for the success of MSI is still the intuitive design and the technical fidelity of the user interface.

In this paper we address the fundamental question of how to present spatially-related content to users of MSI applications. To this end, in sections 2 to 4 we present and discuss 'Point-to-Discover', 'Creative Histories - the Josefsplatz Experience' and 'WikiVienna' as a series of three exemplary research projects which allow to demonstrate how the combination of strong competences in mobile application development and information visualization leads to significant advances in the field of MSI (for more details we refer to http://msi.ftw.at).

Within each of the project descriptions, research problems and results in two specific areas are highlighted: (1) Mobile HCI research and application development, and (2) the development of the underlying server-side rendering algorithms. The paper ends with a brief summary and an outlook on future work.

\section{Point-to-Discover (p2d)}

Our first example, the project Point-to-Discover (p2d, http://p2d.ftw.at), demonstrates the technical feasibility of mobile spatial telecommunications services. Together with our industrial partners mobilkom austria and Siemens Austria, a mobile operator and a mobile service developer, we have built the software platform necessary for MSI telecommunications services. As at the time of project conduction no commercial telephony handset featured the necessary combination of positioning (GPS) and orientation sensing (compass and 3D accelerometers), we have built a prototype sensor board communicating with a standard mobile phone over Bluetooth. The measured location and orientation is processed at a server-side platform, hosting an environment block model and geo-referenced services.

For our demonstrator we chose a mobile restaurant service for the inner city district of Vienna, enabling people to walk through an area of about $3 \mathrm{~km}^{2}$ and to get recommendations about restaurants in their vicinity, simply by pointing at them.

Two lines of HCI and visualization-related research were conceived within the p2d project. First, we explored and structured the design space for visually presenting spatially anchored services or points of interests (POIs). Second, we developed a set of spatial selection algorithms enabling the presentation of POIs that are currently visible for the user: the Local Visibility Model (LVis).

\subsection{Design dimensions of MSI applications}

The overarching goal for MSI applications should be to provide an efficient referential relationship between the virtual and the real world [8] . For example, if a user is looking for a pizzeria in the vicinity, the p2d application should present a recommendation such that the referenced restaurant can easily be found by the user. But how should such an interface look like? To properly address this challenge, decisions within a multidimensional design space are necessary. The most important dimensions can be sketched as follows:

Orientation-awareness. Orientation-awareness describes the capability of phones with spatial sensors such as compasses to rotate the display in alignment to the physical surroundings, so that what is in front of the user is displayed on the top of the display. From a cognitive perspective, users should be supported by orientationaware displays, because they can directly match the virtual information with its real counterpart. Previous empirical research has partly substantiated this hypothesis, at least for indoor navigation [9] . Our comparative user study with a Wizard-of-Oz prototype showed that POIs are much easier accessible with orientation-aware displays than with a standard static version [6] .

Representation of environmental objects. An important consideration is how to represent surrounding objects. Whereas theoretically, a photorealistic mapping may be most desirable, there are several reasons to also take simpler representations into account. In order to achieve a maximum of efficiency, only the information relevant for POI access should be shown to the user (e.g. which object is currently selected), other details may even be distracting. Furthermore, mobile phones will in the foreseeable future impose constraints on the fidelity of graphic rendering. We thus designed two relatively simplistic environmental representations of the surroundings: a 2D billboard model and a 3D block model (see Figure 2). Preliminary results indicate that 2D billboard representations are not satisfactory, but that 3D block representations are very efficient in terms of POI access, and are difficult to be surpassed by more realistic representations with textures.

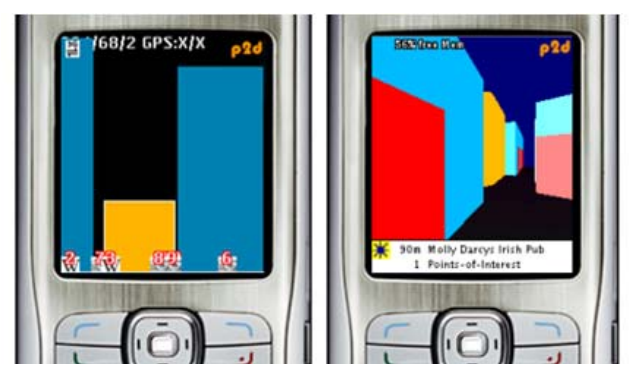

Figure 2: 2D billboard model (left) and 3D block model (right) 
Perspective. Conceptually, users could be confronted from several perspectives with virtual representations of their visible environment and their nearby POIs. Of course, if a direct match between surroundings and virtual representation is desired, the first-person view from a horizontal perspective is preferable (see (1) in Figure 3 ). However, a more elevated perspective could provide a better overview of the surroundings. This could either be realized by the bird's eye perspective (2) or a vertical view (3), as known from maps. Our informal investigations have so far not yielded a clear preference in this regard.

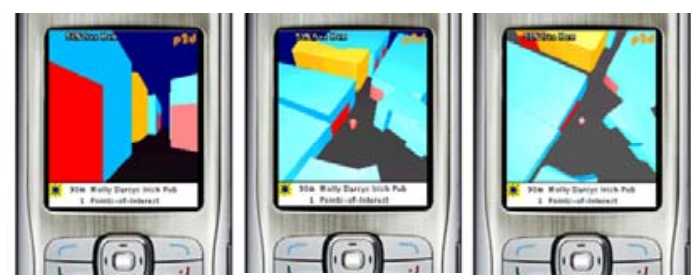

Figure 3: First person view (1), bird's eye perspective (2) and vertical view (3)

Field of view. Another question of interest is how much of the surrounding terrain should be shown to the user (Figure 4). It is still a matter of empirical research whether this should be the whole surrounding scene, or only the portion that is visible in front of the user. First experiences within the project, however, indicate that the field of view should not be restricted, simply because pedestrians often turn around, which would otherwise result in objects popping in and out of the screen.

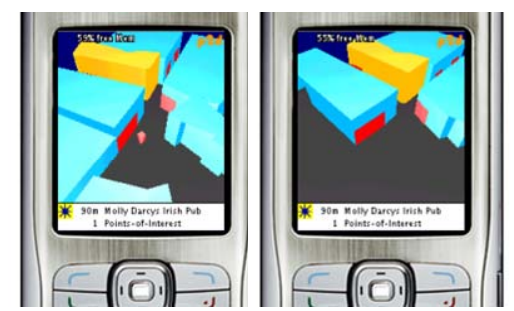

Figure 4: Different fields of view

\subsection{The Local Visibility Model}

In contrast to traditional geospatial search queries returning point-shaped references to geo-coded content, a specialized query engine considering a user's actual field of view had to be developed for p2d. This Local Visibility Model (LVis) not only contains the visible POIs but also an abstraction of the surrounding's actual geometry formulated in XML.

Information that is related to objects, geographical features or buildings that are visible to the user may be contextually more relevant than information about ob- jects that are not. Interactive, ego-centric maps on mobile devices provide added value if they contain indications of the visibility of surrounding features, as has been shown by [6] Visibility information enables entirely new interaction metaphors on mobile devices equipped with advanced sensors such as GPS and digital compass, as has been described by [2] and [15] .

\section{Creative Histories}

The Creative Histories project has been started in order to create a digital version of a set of historic buildings and their contained artifacts, namely the square Josefsplatz in Vienna. One of the challenges of the project was to provide intuitive access to the digital recreation of these buildings through a number of historic epochs.

Based on the reconstruction of the architecture and artifacts, the project presented a host of additional information, such as videos, texts and audio files in the form of a virtual walkthrough. Specialized viewer applications for both a desktop and mobile devices provide simple navigation through the $3 \mathrm{D}$ models and the associated media files. An important aspect of this project was the conceptual simplification of this large information space, such that it can be presented to the user in a comprehensible form even on limited mobile devices.

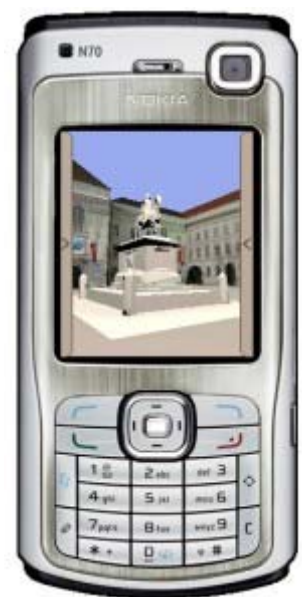

Figure 5: 3D representation of Vienna's Josefsplatz

\subsection{A window to the past}

From a mobile HCI perspective, we were interested in the multidimensional exploration of the surrounding space, using the above-described spatially aware mobile sensor prototype. Going beyond the pure POI access focus in $\mathrm{p} 2 \mathrm{~d}$, we developed a mobile exploration technique that turns the phone screen into a 'window to the past' [4] . This encompasses a historically accurate 3D 
model of the site and its monuments, viewed exactly from the user's current real world position and orientation. Looking for instance towards the Austrian National Library at Vienna's Josefsplatz and aiming the mobile device in the same direction allows the user to sweep back in time to experience how this building has changed during the last decades. After adding appropriate small icons to the rendered 3D model, linked to various kinds of documentary such as sound, speech, text, and video, the user is able to experience a multimodal presentation of Vienna's cultural heritage (see Figure 5: ).

\subsection{Managing rendering data for highly het- erogeneous devices}

Most current rendering and modeling applications and geometric reconstruction methods are designed for modeling large and detailed models for consumption on ever more powerful desktop computers or game consoles. Due to our intention of providing a geometric representation of the Josefsplatz on both the desktop and on mobile devices [16] , it was necessary to develop adequate methods for simplifying the highly complex geometric models while retaining the correct meta-data associations.

In cooperation with the OeFAI, the Austrian Research Institute for Artificial Intelligence, we developed an adequate data representation that accommodates these widely diverging requirements for geometric models. Thus the Creative Histories project has demonstrated that, despite of the limitations of the graphical capabilities of mobile devices, appropriate geometric simplification methods can be used to provide geometric models that retain a large part of the contextual and semantic information available in the full models. Note, however, that the actual geometric model had to be created by specialists and was therefore fairly resource intensive.

\section{WikiVienna}

The ongoing project 'WikiVienna' consequently continues the work presented so far. WikiVienna enables the user to create a detailed 3D model of her home town by contributing photos of buildings via her mobile phone. The model is further enriched with locative media und semantic information. Participants steadily augment the model with multimedia content (photos, audio and video) recorded and submitted via a client application on their mobile phones which are also used as the interface to the user-generated georeferenced content. The goal of the project is to build up a medium for city exploration and to provide interaction via mobile phones with this information space. The resulting framework is applied to the city of Vienna.

WikiVienna's strong visualization aspect and its focus on mobile phones differ from related work studying the attachment of digital data to real world locations (e.g. [10] [11] ). The availability of an extensive, detailed 3D urban model offers completely new visualization possibilities on mobile devices for e.g. guidance and representation of geo-referenced content. Apart from displaying realistically looking $3 \mathrm{D}$ model snippets on the mobile phone, an appropriate server-side rendering engine can provide on-the-fly calculated images beyond common maps in vertical view. Further on, the applied techniques will even enable augmented reality applications on mobile phones where the video stream of a built-in camera is overlaid with geo-coded information.

WikiVienna pools the experiences and results gathered in p2d and Creative Histories. In combination with a powerful server-side reconstruction and rendering service, the project is going to demonstrate manifold novel visualization possibilities for the representation of geocoded content on mobile phones.

\subsection{Advanced device-aware visualization}

As WikiVienna's mobile client is aimed at a mass audience, one of the major technical challenges is to enable all of the features for mass market phones and, at the same time, offer novel visualization and interaction possibilities for high-end devices. The system's deviceawareness is supposed to ensure the best user experience possible using a specific mobile phone.

Device-aware content presentation. Whereas in the p2d project a simplified urban block model with cylindrical buildings (the building's area elevated by its average height) was applied and in 'Creative Histories' a certain square in Vienna was fully modeled in 3D, WikiVienna comprises an extensive urban 3D model. In order to make use of this model on mobile phones and thus take a step towards more realistic 3D representations of geo-referenced content, a server-side simplification of the requested part of the model has to be performed. This device-aware adaption reduces the count of necessary polygons, thus enabling a smooth rendering on different mobile phones. The underlying model enables a variation of the degree of realism: a user might e.g. request a shaded or a textured version, resulting in different file sizes. Alternatively, a device-adequate rendering could be applied transparently to the user.

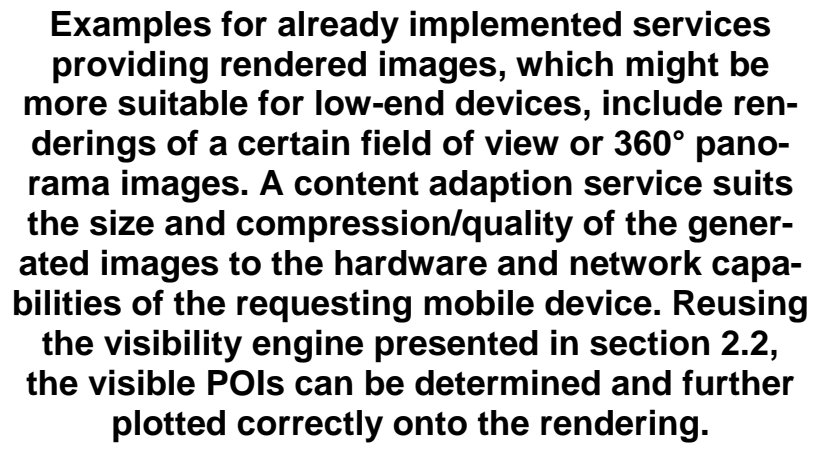

Figure 6 shows an exemplary shaded panorama view (rendered with a virtual whereabout at Michaelerplatz, a square in Vienna's first district) augmented with selectable symbols visualizing available referenced multime- 
dia content. Again, the degree of realism is customizable but does not significantly affect the size of the transferred data.

A prototypical panorama viewer for mobile phones has been developed in order to experiment with different input types depending on the phone's technical features. On low-end devices the panorama may be rotated using the numerical or cursor keys. If the mobile phone is equipped with acceleration sensors the view is adapted to the measured tilt. Finally, a digital compass enables a really intuitive orientation rotating the panorama according to the user's turn. Independent from the chosen type of interaction, the POI closest to the display center is dynamically selected and can be activated pressing a certain key.

\section{Applying the visibility engine introduced above, an augmented reality view is going to be imple- mented for high-end-devices equipped with GPS, compass and acceleration sensors, to conclude the different visualization possibili- ties. The vision of a switchable content repre- sentation is depicted in}

Figure 6 using a shaded rendering and an augmented reality panorama view. Related work dealing with augmented reality on mobile phones mainly relies on visual markers like matrix barcodes as orientation point for the overlaid information (see for instance [13] [14] ]), whereas first steps towards pure sensor-based augmented reality on mobile phones were taken in [11]

With the opportunities of WikiVienna's 3D object rendering and the augmented reality views, we will be able to widen our understanding of the MSI design space. An important related HCI research issue is the identification of the extent to which the augmented reality view improves the access of nearby POIs, as compared to the simple 3D block models investigated in the p2d project. A related design issue is the additional information required for orientation (e.g., street names, directions) that needs to be superimposed over the reality layer.

High-precision device-dependent content annotation. In order to correctly anchor the contributed media in the information space, the location of the mobile device (where the recording has been taken) has to be submitted to the server as well. If the device is not equipped with a GPS receiver and hence, the location cannot be determined by the mobile device itself (as is currently still the case with most mobiles), a server-side network-based localization method is used. Since the resulting inaccuracy may amount to a few hundred meters in urban environment, whereas the precision of the geo-references of the content is crucial for the overall system quality, we plan to offer a precision control based on the available urban model. The range of inaccuracy can be used to select the set of surrounding buildings which then is displayed in a device-accurate manner, e.g. in a $3 \mathrm{D}$ view, on the mobile phone. Pressing the phone's numerical keys the user may switch between the objects (e.g. highlighting or magnifying the active building) and finally select the one she wants the recording to be attached to. In this manner, no verbose text input like address information to specify the location is needed: only a few intuitive keystrokes are necessary to precisely spatially annotate the user-generated data. Such an object-oriented selection is not possible with commonly used static map images lacking of semantic information. Further position and object selection possibilities enabled by the underlying urban model are being evaluated.

\subsection{D reconstruction}

As already mentioned, in the sketched collaborative reconstruction effort participants supply and upload images to the server. In order to provide an adequate geometric model based on these images, it is necessary to enhance and modify the current reconstruction algorithms.

Most of these algorithms have been developed for use with high-quality cameras that can be elaborately calibrated for the reconstruction task. The participants in WikiVienna will use a wide variety of cheap cameras to provide data for reconstruction of the buildings in the central part of Vienna. In order to achieve the necessary quality in the resulting geometric models we are working on methods that use a simplified model provided by the city of Vienna as a basis, and use various geometric reconstruction methods in order to hierarchically reconstruct geometric detail on these simple models.

\section{Conclusions}

This paper has dealt with an integrated (and interdisciplinary) approach for investigating future mobile services. We have seen that the strong current trend of enhancing mobile devices with advanced navigation sensor technology like built-in GPS receivers, digital compasses and acceleration sensors will open the path towards highly intuitive interaction between the user and her physical surrounding, but at the same time has to be complemented by state-of-the art rendering technology on the server side. Therefore, we have argued for a joint approach integrating MSI and visualization, whose potential has been demonstrated with three consecutive application-oriented projects exploring different ways of representing the user environment on the mobile device

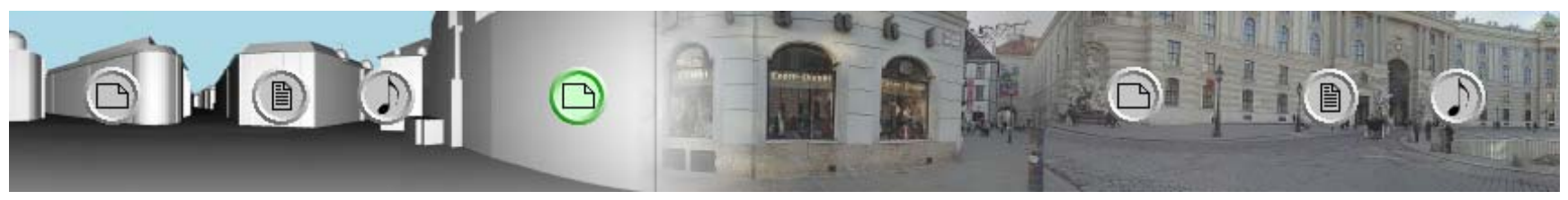


in order to allow for efficient and intuitive modes of user interaction.

Whereas these case studies have shown that visualization is clearly one of the central issues in this context, our current MSI research activities go far beyond and additionally strive for integrating also audio and gesturebased approaches as further important features and options for a natural mode of user interaction. For example, within our focus on device-awareness, we are looking into possibilities of controlling orientation by tiltsensors only, thus alleviating the requirement of having a compass embedded in a certain device.

\section{Acknowledgements}

This paper has been partially funded within the Austrian Kplus and COMET frameworks, the Vienna Science and Technology Fund (WWFT), mobilkom austria AG, and Siemens Austria AG. The authors would like to thank their colleagues Rainer Simon and Stefan Maierhofer for many helpful discussions.

\section{References}

[1] ProgrammableWeb. http://www.programmableweb.com/mapping

[2] Max J. Egenhofer. Spatial Information Appliances: A Next Generation of Geographic Information Systems. First Brazilian Workshop on GeoInformatics. 1999.

[3] Peter Fröhlich, Rainer Simon and Lynne Baillie. Introduction to the special issue on Mobile Spatial Interaction. To appear in: Personal and Ubiquitous Computing Journal. 2008.

[4] Lynne Baillie, Harald Kunczier and Hermann Anegg. Rolling, Rotating and Imagining in a Virtual Mobile World. Proceedings of MobileHCI. 2005.

[5] Gerhard Schall, Erick Mendez, Ernst Kruijff, Eduardo Veas, Sebastian Junghanns, Bernhard Reitinger and Dieter Schmalstieg. Handheld Augmented Reality for Underground Infrastructure Visualization. Special Issue on Mobile Spatial Interaction, Journal on Personal and Ubiquitous Computing. 2008.
[7] Ric Ferrardo. The Year of Location-Based Services (LBS). Ric Ferrardo’s blog, Friday, February 15, 2008. http://mobverge.blogspot.com/2008/02/year-of-locationbased-services-lbs.html

[8] Annti Oulasvirta, Sara Estlander and Annti Nurminen. Embodied interaction with a 3D versus 2D mobile map. Accepted for: Journal of Personal and Ubiquitous Computing, Special Issue on Mobile Spatial Interaction. 2008.

[9] Fabian Hermann, Gerald Bieber and Antje Duesterhoeft. Egocentric Maps on Mobile Devices. Proc. International Workshop on Mobile Computing. 2003.

[10] Per Persson, Fredrik Espinoza, Petra Fagerberg, Anna Sandin and Rickard Cöster. Geonotes: a location-based information system for public spaces. Designing information spaces: the social navigation approach. Springer, London. 2003.

[11] Giles Lane. Urban tapestries: Wireless networking, public authoring and social knowledge. Personal Ubiquitous Computing, vol. 7. 2003.

[12] Markus Kähäri and David J. Murphy. Mobile Augmented Reality Applications. (2006). URL: http://research.nokia.com/research/projects/mara/index.ht $\mathrm{ml}$

[13] Daniel Wagner. Handheld Augmented Reality. Dissertation, Institute for Computer Graphics and Vision, Graz University of Technology. 2007.

[14] Anderes Henrysson. Bringing Augmented Reality to Mobile Phones. Dissertation, Linköping University, Department of Science and Technology. 2007.

[15] Rainer Simon, Peter Fröhlich and Peter Anegg. Beyond Location Based - The Spatially Aware Mobile Phone. Proceedings of the 6th International Symposium on Web and Wireless Geographical Information Systems (W2GIS 2006), Hong Kong, China, December 4-5, 2006. Springer LNCS, vol. 4295/2006, pp. 12-21.

[16] Robert F. Tobler, Stefan Maierhofer, Konrad Karner and Mario Sormann. Creative Histories - The Josefsplatz Experience, The e-volution of Information Communication Technology in Cultural Heritage. Project Papers of the CIPA/VAST/EG/EuroMed 2006, Nicosia, Cyprus. November 2006.

[17] Gregory D. Abowd, Christopher G. Atkeson, Jason Hong, Sue Long, Rob Kooper and M. Pinkerton. Cyberguide: a mobile context-aware tour guide. Wireless Networks, vol. 3, no. 5, pp. 421-433, 1997.
[6] Peter Fröhlich, Rainer Simon, Lynne Baillie and Hermann Anegg. Comparing conceptual designs for mobile access to geo-spatial information. Proceedings of the 8th conference on Human-computer interaction with mobile devices and services. 2006. 